\title{
Plasma Etching Transfer of a Nanoporous Pattern on a Generic Substrate
}

\author{
L. Menon, ${ }^{\mathrm{a}, \mathrm{z}}$ K. Bhargava Ram, ${ }^{\mathrm{a}}$ S. Patibandla, ${ }^{\mathrm{a}}$ D. Aurongzeb, ${ }^{\mathrm{a}}$ M. Holtz, ${ }^{\mathrm{a}}$ \\ J. Yun, ${ }^{\mathrm{b}}$ V. Kuryatkov, ${ }^{\mathrm{b}}$ and K. Zhu ${ }^{\mathrm{b}}$ \\ ${ }^{a}$ Department of Physics, ${ }^{b}$ Department of Electrical Engineering, and Nano Tech Center, Texas Tech \\ University, Lubbock, Texas 79409, USA
}

\begin{abstract}
We describe a nonlithographic nanofabrication method for creating a nanoporous pattern on any substrate. The approach utilizes plasma etching through a nanoporous template to transfer the pore pattern onto the substrate. We demonstrate this method to transfer a porous alumina pattern consisting of a hexagonal array of $50 \mathrm{~nm}$ diam pores onto an aluminum layer. A nanoporous alumina template $(0.6 \mu \mathrm{m})$ is initially created by electrochemical anodization of an aluminum film $(1 \mu \mathrm{m})$ deposited on a substrate. Controlled plasma etching is then used to etch through the pores onto the aluminum layer below the pores. In this manner, we demonstrate the hexagonal array of $50 \mathrm{~nm}$ diam pores in the aluminum film.

(C) 2004 The Electrochemical Society. [DOI: 10.1149/1.1759973] All rights reserved.
\end{abstract}

Manuscript submitted October 8, 2003; revised manuscript received January 3, 2004. Available electronically June 4, 2004.

Nanoporous alumina is highly effective in the fabrication of arrays of nanometer-scale structures. ${ }^{1-3}$ An aluminum oxide membrane is produced consisting of cylindrical arrays of nanosized pores with diameters ranging from $\sim 10-200 \mathrm{~nm}$. When prepared under special conditions, the pores can exhibit a very high level of ordering., ${ }^{4,5}$ These two factors, small feature size and high level of ordering, make nanoporous alumina an attractive template for patterning in nanofabrication. This approach has already been used to prepare self-assembled nanoarrays of various materials by direct electrodeposition of the material into the pores. ${ }^{6,7}$ This has allowed both detailed investigation of the structure of the nanoarrays and the study of fundamental phenomena at the nanometer scale. However, application of electrodeposited nanostructures in devices is limited, mostly due to poor sample quality of electrodeposited nanostructures. Another problem is that the membrane is commonly grown on commercially available aluminum foil, which limits the range of applicability in devices.

Ideally, one would like to transfer or grow the membrane on commercially viable substrates or epitaxial layers, such as $\mathrm{Si}$, and develop processing methods to offer various device fabrication possibilities. Several attempts have been made in this regard. Most of these attempts have involved physical transferring of the nanoporous alumina template onto a substrate (the "mask" method). The nanoporous alumina template then acts as a mask through which appropriate materials can be grown inside the pores. ${ }^{8-10}$ Although the mask method is useful for fabrication of a self-assembled nanoarray, the area over which these nanoarrays can be grown is very small, only of the order of a few millimeters. This is because the mask method involves thinning of the alumina template to a thickness of the order of 0.2-0.5 $\mu \mathrm{m}$. Aluminum oxide, being brittle, breaks up into small pieces, of the order of a few millimeters at such a small thickness. Hence, instead of transferring the membrane, one would like to "grow" the membrane directly on a substrate. In this paper we present an approach that allows us to grow porous alumina membranes directly on a substrate. The method also allows us to transfer the nanopore pattern onto the substrate, which in turn increases the versatility of the method.

The technique combines an anodization process to produce template arrays and plasma etching to reveal an intermediate layer, a seed/adhesion layer, or the substrate. By anodizing an aluminum film, a thin nanopore array is first created on a substrate. A major problem for applications based on this method is posed by the etching of the thin barrier layer of alumina at the pore bottoms to pattern layers directly below. Wet chemical etching methods, such as the use of dilute phosphoric acid, although attempted in the past by other research groups, ${ }^{11,12}$ are not particularly useful. In addition to re- moving the barrier layer, the wet etching also causes pore widening, thus increasing the pore diameter and in some cases completely removing the alumina film. The directional and anisotropic attributes of plasma etching are essential for this approach, because the template pores are small with very high aspect ratios. The etching conditions require critical control to avoid complete etching of the membrane. In this paper, we describe chlorine plasma etching experiments for producing pores that are tens of nanometers in diam and appear in an ordered hexagonal array.

Silicon wafers were used as the starting substrate material for demonstrating the technique. In principle, the approach can be extended to other substrates, and we have conducted preliminary experiments on epitaxial GaN. ${ }^{13}$ Substrates are prepared using an acetone/ethanol deionized water clean, then loaded into an E-beam evaporator with base pressure $\sim 10^{-7}$ mTorr. A $5 \mathrm{~nm}$ thick adhesion layer of $\mathrm{Ti}(99.98 \%$ purity starting material) is deposited on the $\mathrm{Si}$ substrates by E-beam evaporation followed by a $1 \mu \mathrm{m}$ thick layer of $\mathrm{Al}$ (99.999\% purity) without breaking vacuum. Without the Ti layer, the subsequently grown membrane peels from the substrate. The aluminum film is then anodized in an acid under de conditions. During anodization, aluminum is converted to nanoporous aluminum oxide. More details of the template process can be found

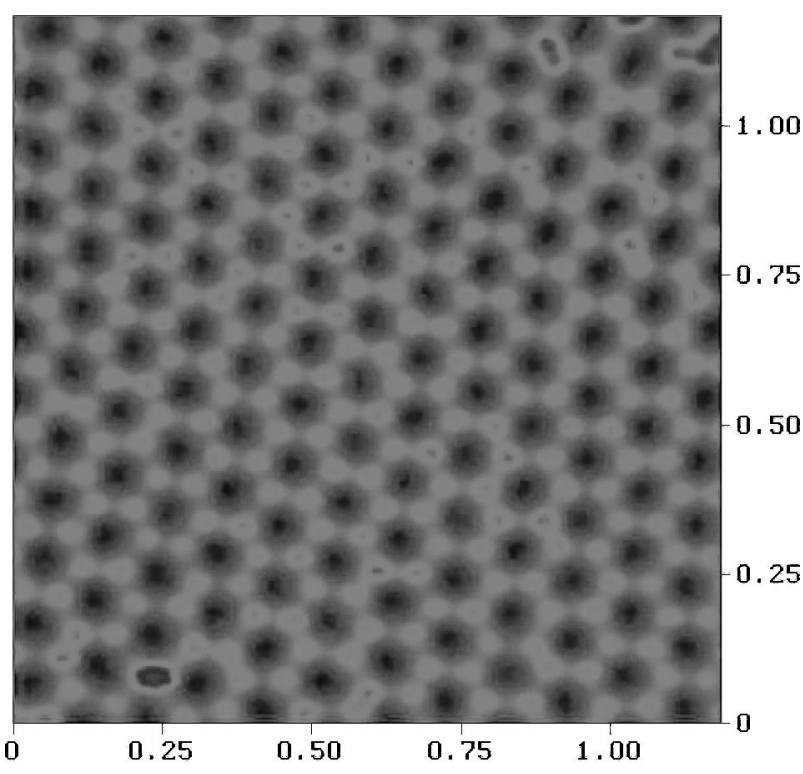

UM 

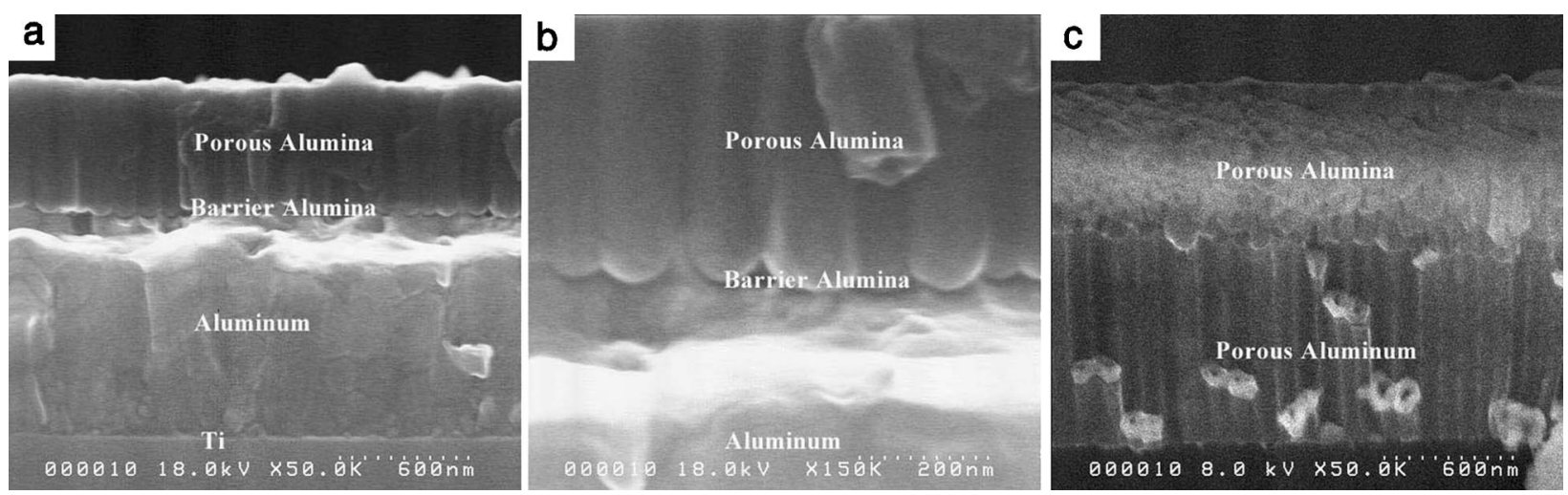

Figure 2. (a) SEM cross-sectional image of as-deposited template. (b) Magnified view of the same. (c) SEM cross-sectional image following $60 \mathrm{~s}$ etch with 200 W RIE power.

elsewhere. ${ }^{1}$ The pore diameter is controlled by the anodization conditions. Anodization in $3 \%$ oxalic acid at $40 \mathrm{~V}$ gives a membrane with pore diam $50 \mathrm{~nm}$, as addressed here. Figure 1 shows a representative atomic force microscope (AFM) image demonstrating the regularity in pore diameter and the obtained hexagonal array.

A scanning electron microscope (SEM) cross-sectional image of the as-deposited template is shown in Fig. 2a. Clearly seen are the Ti adhesion layer, the Al layer that remains following anodization, and the alumina layer produced by the anodization process. The presence of a thin barrier layer of alumina (U-shaped barrier) is also seen at the pore bottoms. The thickness of the barrier layer is only of the order of $10-20 \mathrm{~nm}$. The depth of the pores in the membrane is directly proportional to the anodization time. Here the anodization was carried out for 2-3 min, producing a porous alumina template $\sim 0.6 \mu \mathrm{m}$ thick atop the $1.0 \mu \mathrm{m}$ aluminum layer, indicating an expansion in volume. ${ }^{4}$ A magnified view is seen in Fig. $2 b$ showing a SEM image focusing on the barrier layer in the as-fabricated porous alumina.

Plasma etching was carried out using a commercial inductively coupled plasma (ICP) system with reactive ion etching (RIE). $\mathrm{Cl}_{2}$ diluted with $\mathrm{Ar}$ was used as etchant. The basic etching conditions are given in Table I. The low chamber pressure produces conditions of high mean-free path and high Knudsen number. These conditions are conducive to highly directional and anisotropic etching of the high aspect ratio $(\sim 30: 1)$ features. Samples were attached to quartz wafers and loaded into the plasma reactor, where they were held to the chuck by helium flow. The wafer temperature was not controlled; we estimate it to remain below $100^{\circ} \mathrm{C}$ during the etch process. The RIE power was varied from 100 to $300 \mathrm{~W}$. We expect the RIE power to be a critical parameter, because RIE has the combined effect of redirecting etchant adhered to the top surface into the pores and of increasing the volatility of etch by-products. Under these processing conditions, we find $\mathrm{Al}$ to etch at $\sim 2 \mu \mathrm{m} / \mathrm{min}$. The alumina barrier etches in 30-40 s, for an approximate etch rate of 60 $\mathrm{nm} / \mathrm{min}$. Under identical conditions, sapphire is completely etch resistant in $60 \mathrm{~s}$. Although the addition of $\mathrm{BCl}_{3}$ etches sapphire, ${ }^{15}$ this approach is undesirable here because the alumina template would etch more effectively, possibly eliminating the template. Based on the selectivity of chlorine etching to Al over alumina, the correct etching conditions are expected to redirect etchant into the nanopore

\begin{tabular}{lc} 
Table I. Basic etching conditions. & \\
Plasma parameter & Value \\
\hline ICP power & $300 \mathrm{~W}$ \\
RIE power & $100-300 \mathrm{~W}$ \\
Chamber pressure & $8 \mathrm{mTorr}$ \\
$\mathrm{Cl}_{2} /$ Ar flow rates & $20 / 5 \mathrm{sccm}$
\end{tabular}

template, break through the barrier layers of alumina, and rapidly etch the aluminum, leaving the alumina template largely intact. Furthermore, charging of the insulating alumina results in an appreciable field at the pore opening, because ions are expected to be readily captured near the surface due to the small pore sizes. ${ }^{16}$ The resultant field assists channeling of ions into the high aspect ratio features.

The etch process is highly sensitive to the etch time because of the relative etch rates of $\mathrm{Al}$ and alumina. For example, etching for $30 \mathrm{~s}$ at $200 \mathrm{~W}$ RIE power is not sufficient to break through the alumina barrier layer, although there is a reduction in total thickness of the porous alumina layer at the top $(0.3 \mu \mathrm{m})$. However, a $60 \mathrm{~s}$ etch at the same $200 \mathrm{~W}$ RIE power is sufficient to break through the barrier layer. Figure 2c shows SEM cross-sectional images of the sample following a $60 \mathrm{~s}$ etch with $200 \mathrm{~W}$ RIE power. The pores in the top nanoporous alumina layer can be clearly seen, and the alumina layer at the top is further reduced to be about $0.2 \mu \mathrm{m}$ thick. In addition, the etch has penetrated through the barrier region and continued down through the $\mathrm{Al}$ to the $\mathrm{Ti}$ adhesion layer. Several of the Al nanotubes produced by the process have snapped from cleaving, showing the hexagonal shape of the parent hole template. This result

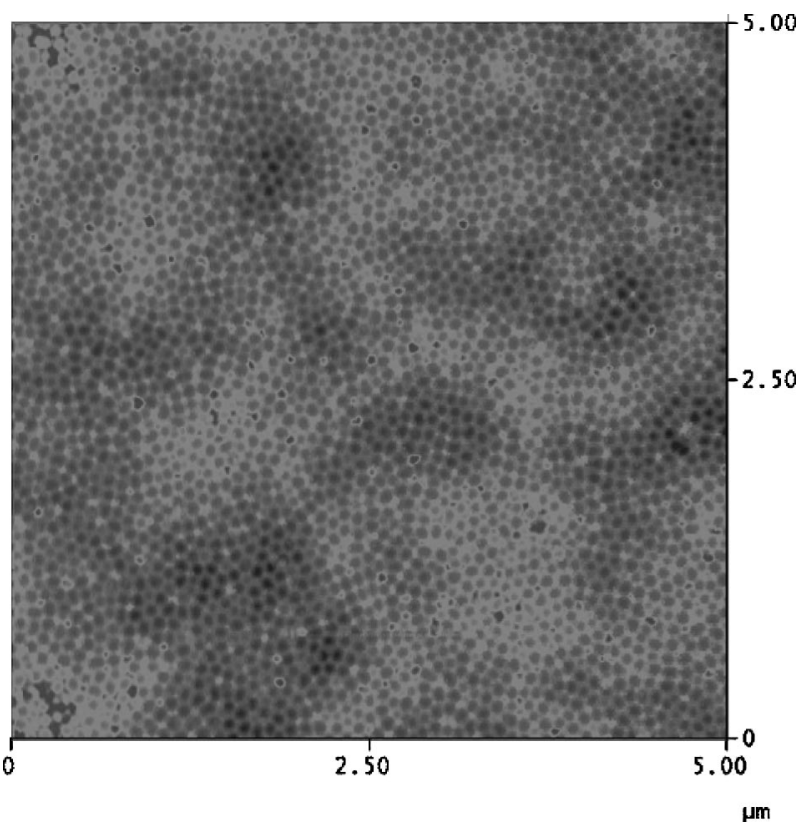

Figure 3. AFM image of top view of the aluminum template post removal of the porous alumina layer (pore diam $\sim 50-60 \mathrm{~nm}$ ). 
shows that these conditions produce the highly directional and anisotropic etching necessary to reach the adhesion/substrate level for extremely small features.

The etch result was also sensitive to the RIE power. For example, a $60 \mathrm{~s}$ etch at a reduced $100 \mathrm{~W}$ RIE power is not sufficient to break through the barrier layer. Evidently, the roles of RIE to redirect etchant deep into the nanopores and to assist desorption of byproducts is essential. In contrast, at a higher RIE power of $300 \mathrm{~W}$, we observed $\sim 200 \mathrm{~nm}$ removal of the alumina surface and significantly degraded morphology. Under these conditions, a $60 \mathrm{~s}$ etch did not break through the barrier layer. We suggest from this that the alumina template is physically etched (i.e., sputtered) at this RIE power. The associated surface damage sufficiently restricts the transfer of etchant into the nanopores, thereby eliminating the barrier etch.

Following breakthrough of the barrier layer, the etch time dictates the depth of the aluminum pores, whether or not the Ti layer is etched, and the extent of etching into the substrate. Thus, the etch can be used to reveal several possible array structures with high regularity for subsequent process steps. Alumina surviving the etching process can be stripped away to reveal the $\mathrm{Al}$ template by preferential wet etching. Figure 3 shows an AFM image of the top view of the aluminum template post removal of the porous alumina layer by soaking in a mixed solution consisting of $0.2 \mathrm{M}$ chromic/0.4 M phosphoric acid. The pore diam is of the order of 50-60 nm, similar to that of the initial alumina film. We demonstrate from this that the alumina pore pattern is directly transferred to the aluminum with no significant degradation.

\section{Conclusion}

We have developed an approach for producing nanometer-scale pore patterns of aluminum on silicon substrates. Pore sizes as small as $50 \mathrm{~nm}$ were plasma etched with aspect ratios of $\sim 30$. The etch process requires critical control over the etch time due to differing rates of $\mathrm{Al}$ and alumina etch. We observed a strong dependence of the etch on the RIE power. The process is highly versatile and appears to be restricted only by the need to produce the patterning template from $\mathrm{Al}$ and alumina.

\section{Acknowledgment}

The authors acknowledge partial support for this work from the National Science Foundation (ECS-0070240, ECS-0323640, ECS0304224, and CTS-0210141).

Texas Tech University assisted in meeting the publication costs of this article.

\section{References}

1. L. Menon, in Quantum Dots and Nanowires, H. S. Nalwa and S. Bandopadhyay, Editors, p. 141, American Scientific Publishers, Stevenson Ranch, CA (2003).

2. D. Routkevich, A. A. Tager, J. Haruyama, D. Almawlawi, M. Moskovits, and J. M. Xu, IEEE Trans. Electron Devices, 43, 1646 (1996).

3. M. Moskovits and B. Schmid-Halter, Int. Pat. Wo88/02538 (1988).

4. H. Masuda and K. Fukuda, Science, 268, 1466 (1995).

5. H. Masuda, F. Hasegawa, and S. Ono, J. Electrochem. Soc., 144, L127 (1997)

6. M. Zheng, L. Menon, H. Zeng, Y. Liu, S. Bandyopadhyay, R. D. Kirby, and D. J. Sellmyer, Phys. Rev. B, 62, 12282 (2000).

7. L. Menon, M. Zheng, H. Zeng, S. Bandyopadhyay, and D. J. Sellmyer, J. Electron Mater, 29, $510(2000)$

8. D. Crouse, Y.-H. Lo, A. E. Miller, and M. Crouse, Appl. Phys. Lett, 76, 49 (2000).

9. H. Masuda, K. Yada, and A. Osaka, Jpn. J. Appl. Phys., Part 2, 37, L1340 (1998).

10. J. Liang, H. Chik, A. Yin, and J. Xu, J. Appl. Phys., 91, 2544 (2002).

11. O. Rabin, P. R. Herz, S. B. Cronin, Y.-M. Lin, A. I. Akinwande, and M. S. Dresselhaus, Mater. Res. Soc. Symp. Proc., 636, D4.7.1 (2001).

12. Q. Huang, W.-K. Lye, D. M. Longo, and M. L. Reed, Mater. Res. Soc. Symp. Proc., 636, D 9.49 .1 (2001)

13. L. Menon and M. Holtz, Unpublished.

14. O. Jessensky, F. Müller, and U. Gösele, Appl. Phys. Lett., 72, 1173 (1998).

15. C. C. Mitchell, D. D. Koleske, D. M. Follstaedt, N. A. Missert, K. H. A. Bogart, K. Cross, M. E. Coltrin, and A. A. Allerman, 39th Symposium of the New Mexico American Vacuum Society, p. 15, Albuquerque, NM, April 29-30, 2003.

16. W. N. G. Hitchon, Plasma Processes for Semiconductor Fabrication, p. 131, Cambridge University Press, Cambridge (1999). 